\title{
The Impact of China's Lockdown Policy on the Incidence of COVID-19: An Interrupted Time Series Analysis
}

\author{
Mooketsi Molefi $\left(D,{ }^{1}\right.$ John T. Thakanelo ${ }^{D},{ }^{1}$ Thabo Phologolo ${ }^{D},{ }^{1}$ Shimeles G. Hamda $\left(\mathbb{D},{ }^{1}\right.$ \\ Tiny Masupe $\left(1,{ }^{1}\right.$ Billy Tsima $\left(\infty,{ }^{2}\right.$ Vincent Setlhare $(1),{ }^{2}$ Yohana Mashalla $₫{ }^{3}$ \\ and Douglas J. Wiebe $\mathbb{C}^{4}$ \\ ${ }^{1}$ Public Health Medicine Unit, Department of Family Medicine \& Public Health, University of Botswana, Gaborone, Botswana \\ ${ }^{2}$ Family Medicine Unit, Department of Family Medicine \& Public Health, University of Botswana, Gaborone, Botswana \\ ${ }^{3}$ Biomedical Science Department, Faculty of Health Sciences, University of Botswana, Gaborone, Botswana \\ ${ }^{4}$ Department of Biostatistics, Epidemiology \& Informatics, Perelman School of Medicine, University of Pennsylvania, \\ Philadelphia, USA
}

Correspondence should be addressed to Mooketsi Molefi; molefim@ub.ac.bw

Received 9 June 2021; Accepted 28 September 2021; Published 28 October 2021

Academic Editor: Mihajlo Jakovljevic

Copyright (c) 2021 Mooketsi Molefi et al. This is an open access article distributed under the Creative Commons Attribution License, which permits unrestricted use, distribution, and reproduction in any medium, provided the original work is properly cited.

\begin{abstract}
Background. Policy changes are often necessary to contain the detrimental impact of epidemics such as those brought about by coronavirus disease (COVID-19). In the earlier phases of the emergence of COVID-19, China was the first to impose strict restrictions on movement (lockdown) on January 23rd, 2020. A strategy whose effectiveness in curtailing COVID-19 was yet to be determined. We, therefore, sought to study the impact of the lockdown in reducing the incidence of COVID-19. Methods. Daily cases of COVID-19 that occurred in China which were registered between January 12th and March 30th, 2020, were extracted from the Johns Hopkins CSSE team COVID-19 ArcGIS ${ }^{\circledR}$ dashboards. Daily cases reported were used as data points in the series. Two interrupted series models were run: one with an interruption point of 23 January 2020 (model 1) and the other with a 14-day deferred interruption point of 6th February (model 2). For both models, the magnitude of change (before and after) and linear trend analyses were measured, and $\beta$-coefficients reported with $95 \%$ confidence interval (CI) for the precision. Results. Seventy-eight data points were used in the analysis. There was an $11 \%$ versus a $163 \%$ increase in daily cases in models 1 and 2, respectively, in the preintervention periods $(p \leq 0.001)$. Comparing the period immediately following the intervention points to the counterfactual, there was a daily increase of $2,746 \%(p<0.001)$ versus a decline of $207 \%(p=0.802)$ in model 2. However, in both scenarios, there was a statistically significant drop in the daily cases predicted for this data and beyond when comparing the preintervention periods and postintervention periods $(p<0.001)$. Conclusion. There was a significant decrease the COVID-19 daily cases reported in China following the institution of a lockdown, and therefore, lockdown may be used to curtail the burden of COVID-19.
\end{abstract}

\section{Introduction}

The recognition of a possible outbreak followed the identification of a cluster of cases presenting with a rare type of pneumonia [1]. These cases had both epidemiological and geographical ties to the Huanan seafood market in Wuhan, Hubei province, China $[2,3]$. Samples from these patients later revealed a novel type of coronavirus known as SARS COV-2 [4]. A virus closely associated with those that cause severe acute respiratory syndrome (SARS) and Middle East Respiratory Syndrome (MERS) [5, 6].

The few weeks that followed saw more cases being detected in the Hubei province, with Wuhan city having the highest number of cases [7]. The cumulative incidence 
increased exponentially daily reaching a few hundred in less than three weeks [8]. The situation needed containment especially with evidence of local transmission taking place in neighbouring provinces [9].

On January 23rd, 2020, the government of China imposed a lockdown on Hubei province in an effort to control the spread of the disease [10]. The lockdown resulted in restrictions on movement among residents of the province requiring all to stay indoors during this period. There were mixed reactions to this intervention with some labelling it as extreme especially as social support was not guaranteed $[11,12]$. While China has recently reported a decline in the incidence of COVID-19, it is not clear what impact the lockdown has had on this decline.

This study sought to quantify the impact that China's lockdown policy had in reducing the incidence of COVID19 using interrupted time series methods.

\section{Methods}

Cases of COVID-19 in China as reported daily were extracted from the Johns Hopkins CSSE team ArcGIS dashboard [13] for periods between January 12th and March 30th, 2020. These figures were then matched to China's projected population for 2020, estimated at 1,408 526449 according to the United Nations' population division. The daily occurrences of cases were then calculated and used as data points in the time series analysis using 23rd January as the intervention point (Tables 1 and 2, model 1). Additionally, because we did not expect the impact of the lockdown to start immediately, we modelled the data where we carried forward the date intervention to 6th February (Tables 3 and 4, model 2); the length of time corresponding to the maximum incubation period of 14 days for someone who was exposed on or about 23rd January 2020.

Data were modelled as a single-group interrupted time series analysis without a comparator for both scenarios using the itsa syntax in Stata ${ }^{\circledR}$. The level of change and the trajectory of change following the intervention obtained via ordinary least-squares [14] regression estimates were evaluated yielding $\beta$-coefficients and Newey-West standard errors. The Cumby-Huizinga test for serial autocorrelation using actest, lags in Stata $[15,16]$ was employed as a postestimation command following the OLS regression.

\section{Results}

Seventy-eight data points were used in the series with 11 $(14.10 \%)$ and $25(32.05 \%)$ data points used in the preintervention period in Figures 1 and 2, respectively. In the preintervention phase, Table 1 shows a $11 \%(95 \% \mathrm{CI}=3.95,18.23)$ increase in the occurrence of daily cases while Table 3 shows an astronomical increase of $163 \%(95 \% \mathrm{CI}=110.29,216.06)$. When comparing the period immediately following the intervention versus the counterfactuals for both scenarios, the data showed an overwhelming 2,746\% (95\%CI = $1142.18,4350.70)$ increase in daily cases in Table 1 against the counterfactual while Table 3 data shows a $207 \%$ decline in the occurrence of daily cases; however, the latter was not statistically significant $(p=0.832)$. When measuring the magnitude of change between the pre- and postintervention periods, we obtained a $60 \%(95 \% \mathrm{CI}=25.98,95.51)$ decline and $240 \%(95 \% \mathrm{CI}=167.97,313.01)$ decline in the occurrence of daily cases for Tables 1 and 3, respectively.

\section{Discussion}

Prior to this study, there was a question about the effectiveness of lockdown as a strategy for the containment of the spread of COVID-19 [12]. The findings herein reveal an increasing occurrence of daily cases of COVID-19 in the preintervention period, as at the time there were no restrictions on movement of people and goods across China before the lockdown and so disease transmission continued to flare. This significant increment of new cases daily would potentially have reached epic proportions and had the lockdown intervention not been instituted. This evidence is supported by the rising numbers of daily cases reported when comparing the postintervention cases and the counterfactual scenarios.

There was a statistically significant predicted rise in the number of daily cases in model 1 (Table 1) in the period immediately following the intervention. This was to be expected as at the time there may have been many other people who may have been infected and were yet to be diagnosed, but more importantly, the effects of such an intervention would take time to show, hence our modelling of a deferred intervention point. When comparing the preintervention and postintervention periods, while a nondeferred intervention point (Table 1) showed a modest decrease of $60 \%$ in the occurrence of daily cases, Table 3 modelled on deferred intervention point shows an overwhelming 4 times the decline in daily cases. This reduction thus indicates that the lockdown policy not only had a positive impact in reducing the incidence of COVID-19 but also resulted in an accelerated way of reversing the situation. The finding that China's COVID-19 incidence is decreasing is consistent with findings from other studies $[17,18]$.

A more specific yet less stringent intervention is the notion of social distancing which has been implemented its effects evaluated in a several settings, alongside other interventions. Several studies evaluating the effectiveness of the social distancing policy using the interrupted time series analysis methods have been published [19]. Saki et al. studied the effects of social distancing policy on the incidence and deaths of COVID-19 in Iran. The findings were consistent with ours, where they found a decrease in the number of new cases occurring and a decreasing trend after the implementation of the social distancing policy. Additionally, Alimohamadi et al. also demonstrated the positive effects of the social distancing policy in curbing the incidence and mortality from COVID-19 in Iran. Both studies corroborate the findings from China in the early days of the pandemic. Of note is that the impact estimated herein is only conservative as there was a change in the case-definition of a COVID19 case in mid-February in China from symptoms and a positive test to just symptoms [20]. An action that could 
TABLE 1: Interrupted time series ordinary least-squares regression output at 23rd January 2020 intervention point (model 1).

\begin{tabular}{lcccc}
\hline & $\beta$-Coefficient & Std error & $p$ value & 95\% CI \\
\hline Preintervention & 11.09 & 3.58 & $0.003^{*}$ & $3.95,18.23$ \\
Immediately postintervention vs. counterfactual & 2746.44 & 805.13 & $\leq 0.001^{*}$ & $1142.18,4350.70$ \\
Pre- vs. postintervention & -60.75 & 17.45 & $\leq 0.001^{*}$ & $-95.51,-25.98$ \\
\hline
\end{tabular}

TABLe 2: Postlinear trend regression output from 23rd January 2020 interruption point (model 1).

\begin{tabular}{lcccr}
\hline & $\beta$-Coefficient & Std error & $p$ value & $95 \%$ CI \\
\hline Postintervention linear trend & -49.66 & 15.89 & $0.003^{*}$ & $-81.33,-17.99$ \\
\hline
\end{tabular}

TABLE 3: Interrupted time series ordinary least-squares regression output at 14-day deferred intervention (model 2).

\begin{tabular}{lcccc}
\hline & $\beta$-Coefficient & Std error & $p$ value & $95 \%$ CI \\
\hline Preintervention & 163.18 & 26.54 & $\leq 0.001^{*}$ & $110.29,216.06$ \\
Immediately postintervention vs. counterfactual & -206.95 & 971.17 & 0.832 & $-2142.06,1728.16$ \\
Pre- vs. postintervention & -240.49 & 36.40 & $\leq 0.001^{*}$ & $-313.02,-167.97$ \\
\hline
\end{tabular}

Table 4: Postlinear trend regression output using 6th February 2020 interruption point (model 2).

\begin{tabular}{lcccc}
\hline & $\beta$-Coefficient & Std error & $p$ value & $95 \%$ CI \\
\hline Postintervention linear trend & -77.32 & 24.60 & $0.002 *$ & $-126.34,-28.30$ \\
\hline
\end{tabular}

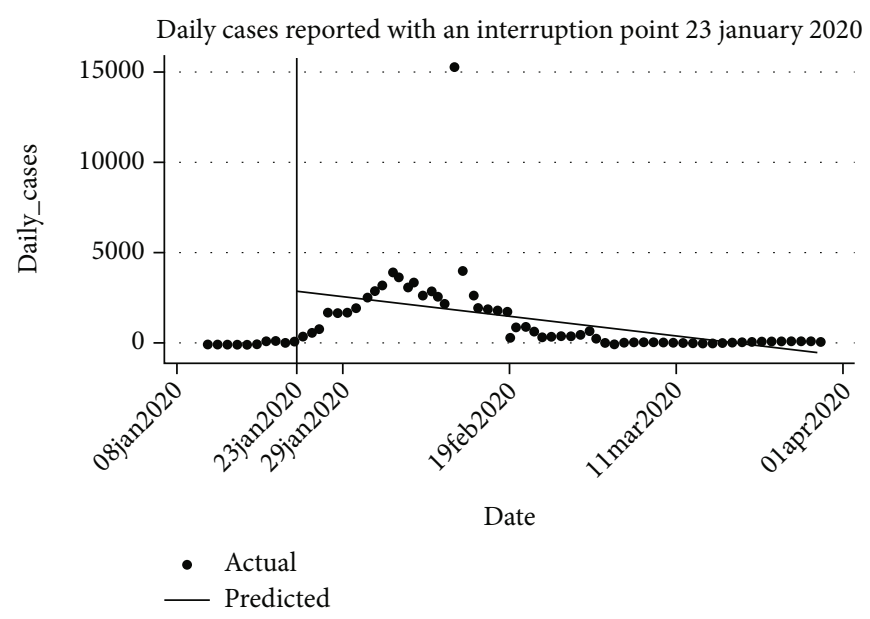

Figure 1: Daily cases reported over time showing a 23rd January interruption point (model 1).

result in the artefactual increment of COVID-19 cases is reported.

Since we were evaluating the impact of a large-scale intervention using data before and after an intervention, interrupted time series methods were suitable for this purpose. These methods are powerful methods of high validity since they control for common threats of confounding which exist in other observational studies [21] therefore are a good compromise when it is not feasible or unethical to randomize units/subjects [22]. With a distinct time of intervention when the lockdown was announced and factoring in an estimated period of diffusion, we believe that approach enabled us to mimic the truth about the evolution of the disease in China. Furthermore, sensitivity analysis revealed the validity of our model up to 4 lags for both scenarios.

As more countries have started having cases of COVID19 , they will be looking for effective strategies to control the 


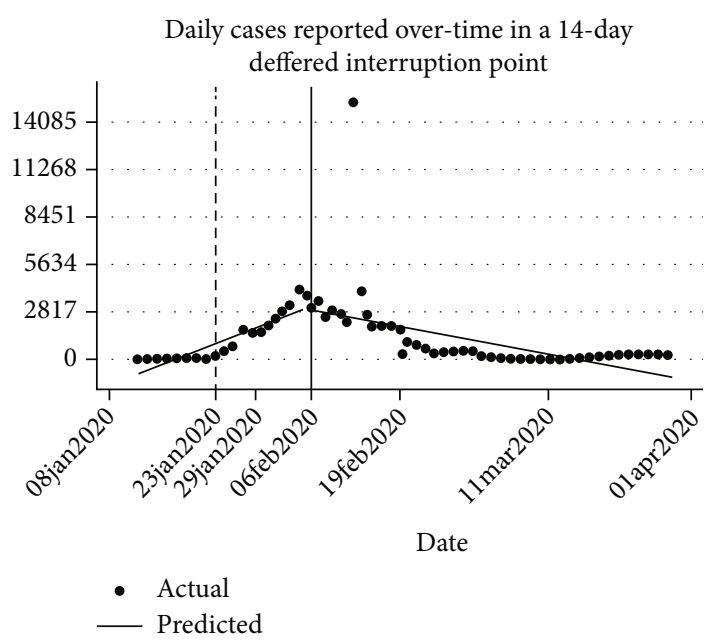

FIGURE 2: Daily cases reported over time showing a 14-day deferred interruption point from 23 January to 6th February 2020 (model 2).

outbreak in their settings. This paper demonstrates the effectiveness of a lockdown strategy and that it may be necessary to prevent more cases from occurring during an outbreak.

There are limitations that need noting in this study. First, we cannot rule-out the possibility of other interventions that could have contributed to the change; though so far, none have been published or reported. Secondly, we relied on secondary data as it was being reported, and we only had limited data points to model with before the intervention as it was only the beginning of the outbreak. A review and analysis of more recent data in other settings to evaluate the impact of lockdown strategy in reducing the burden of COVID-19 cases are needed.

\section{Conclusion}

There is evidence that the lockdown policy introduced by China in containment of COVID-19 has completely reversed the occurrence of COVID-19 cases reported daily. Lockdown policy presents a viable option of burden of COVID-19.

\section{Data Availability}

The data are freely available online at https://www.arcgis .com/apps/dashboards/

bda7594740fd40299423467b48e9ecf6 and the United

Nations' population division website.

\section{Additional Points}

What Is Already Known on the Subject. Evidence for the effectiveness of "lockdown" as a strategy in controlling the surge of COVID-19 was lacking, despite many countries continuing to adopt it. What the Study Adds. This study illustrates the effectiveness of the "lockdown" strategy or policy in controlling the surge in cases of COVID-19 through the use of quasi-experimental methods on early data generated from China. Policy Implications. Lockdown policy or strategy could be adopted as an effective means of reducing the COVID-19 incidence. Patient and Public Involvement. Since this is an ecological study looking at aggregate data, it was not possible to involve patients or the public in the design, conduct, and reporting of our research.

\section{Ethical Approval}

No data at individual patient level were collected; thus, no ethical approval nor individual consent was applicable.

\section{Disclosure}

This paper is already published in the preprint given in the below link https://www.researchsquare.com/article/rs$32944 / v 1$.

\section{Conflicts of Interest}

The authors declare that they have no known competing financial interests or personal relationships that could have appeared to influence the work reported in this paper.

\section{Authors' Contributions}

MM conceptualized and designed the study, acquired the data, and analyzed them. JTT, TP, SGH, TM, BT, VS, YM, and DJW assisted with the interpretation, drafting the article, and revising the manuscript critically for important intellectual content. All authors approved the final version to be submitted.

\section{Acknowledgments}

We are thankful to Johns Hopkins University's CSSE team for publishing updates on new cases related to COVID-19 on the ArcGIS ${ }^{\otimes}$ dashboard and the United Nation population division for sharing country-level population data.

\section{References}

[1] N. Zhu, D. Zhang, W. Wang et al., "A novel coronavirus from patients with pneumonia in China, 2019," New England Journal of Medicine, vol. 323, p. 8, 2020.

[2] F. Wu, S. Zhao, B. Yu et al., "A new coronavirus associated with human respiratory disease in China," Nature, vol. 579, no. 7798, pp. 265-269, 2020.

[3] N. Chen, M. Zhou, X. Dong et al., "Epidemiological and clinical characteristics of 99 cases of 2019 novel coronavirus pneumonia in Wuhan, China: a descriptive study," Lancet, vol. 395, no. 10223, pp. 507-513, 2020.

[4] W. Wang, Y. Xu, R. Gao et al., "Detection of SARS-CoV-2 in different types of clinical specimens," Journal of the American Medical Association, vol. 323, no. 18, pp. 1843-1844, 2020.

[5] M. Barry, M. Al Amri, and Z. A. Memish, "COVID-19 in the shadows of MERS-CoV in the Kingdom of Saudi Arabia," Journal of epidemiology and global health, vol. 10, no. 1, pp. 1-3, 2020.

[6] H. M. Ashour, W. F. Elkhatib, M. M. Rahman, and H. A. Elshabrawy, "Insights into the Recent 2019 Novel coronavirus 
(SARS-CoV-2) in light of past human coronavirus outbreaks," Pathogens, vol. 9, no. 3, p. 186, 2020.

[7] T. Singhal, "A review of coronavirus disease-2019 (COVID19)," Indian Journal of Pediatrics, vol. 87, no. 4, pp. 281-286, 2020.

[8] J. T. Wu, K. Leung, and G. M. Leung, "Nowcasting and forecasting the potential domestic and international spread of the 2019-nCoV outbreak originating in Wuhan, China: a modelling study," The Lancet, vol. 395, no. 10225, pp. 689-697, 2020.

[9] S. Zhao and H. Chen, "Modeling the epidemic dynamics and control of COVID-19 outbreak in China," Quantitative biology, pp. 1-9, 2020.

[10] R. Russel, China Puts Wuhan on Lockdown with All Transport Shut in Bid to Contain Virus, 2020, http://www.express.co.uk.

[11] F. Kretschmer, Wuhan Lockdown: China Takes Extreme Measures to Stop Virus Spread, 2020.

[12] A. Gunia, China's draconian lockdown is getting credit for slowing coronavirus. Would it work anywhere else?, 2020, https://www.time.com/health.

[13] [Available from: https://www.arcgis.com/apps/dashboards/ bda7594740fd40299423467b48e9ecf6.

[14] Y. Alimohamadi, K. Holakouie-Naieni, M. Sepandi, and M. Taghdir, "Effect of social distancing on COVID-19 incidence and mortality in Iran since February 20 to May 13, 2020: an interrupted time series Analysis," Risk Management and Healthcare Policy, vol. Volume 13, pp. 1695-1700, 2020.

[15] R. E. Cumby and J. Huizinga, "Investigating the correlation of unobserved expectations: expected returns in equity and foreign exchange markets and other examples," Journal of Monetary Economics, vol. 30, no. 2, pp. 217-253, 1992.

[16] C. F. Baum and M. E. Schaffer, ACTEST: stata module to perform Cumby-Huizinga general test for autocorrelation in time series, 2013, https://sociorepec.org.

[17] H. Lau, V. Khosrawipour, P. Kocbach et al., "The positive impact of lockdown in Wuhan on containing the COVID-19 outbreak in China," Journal of Travel Medicine, vol. 27, 2020.

[18] C. C. Ku, T.-C. Ng, and H.-H. Lin, "Epidemiological benchmarks of the COVID-19 outbreak control in China after Wuhan's lockdown: a modelling study with an empirical approach," 2020, Available at SSRN 3544127.

[19] M. Saki, M. K. Ghanbari, M. Behzadifar et al., "The impact of the social distancing policy on COVID-19 incidence cases and deaths in Iran from February 2020 to January 2021: insights from an interrupted time series analysis," The Yale Journal of Biology and Medicine, vol. 94, no. 1, pp. 13-21, 2021.

[20] R. Wei, "Changes to China's case definition of COVID-19 creates a spike in cases," Pharmaceutical technology, 2020.

[21] S. B. Soumerai, D. Starr, and S. R. Majumdar, "How do you know which health care effectiveness research you can trust? A guide to study design for the perplexed," Preventing chronic disease, vol. 12, 2015.

[22] R. B. Penfold and F. Zhang, "Use of interrupted time series analysis in evaluating health care quality improvements," Academic Pediatrics, vol. 13, no. 6, pp. S38-S44, 2013. 